\title{
Enseigner la sociologie de la culture dans des formations professionnalisantes
}

Entretien avec Lionel Arnaud et Vincent Dubois

Teaching the Sociology of Culture as Part of Professional Training. Interview with Lionel Arnaud and Vincent Dubois

Enseñar la sociología de la cultura en las formaciones profesionalizantes.

Entrevista con Lionel Arnaud y Vincent Dubois

Frédéric Chateigner, Jérémy Sinigaglia, Lionel Arnaud et Vincent Dubois

\section{(2) OpenEdition}

\section{Journals}

Édition électronique

URL : http://journals.openedition.org/bssg/345

DOI : $10.4000 /$ bssg.345

ISSN : $2490-9424$

Éditeur

Presses universitaires de Vincennes

Référence électronique

Frédéric Chateigner, Jérémy Sinigaglia, Lionel Arnaud et Vincent Dubois, « Enseigner la sociologie de la culture dans des formations professionnalisantes », Biens Symboliques / Symbolic Goods [En ligne], 4 | 2019, mis en ligne le 27 juin 2019, consulté le 04 mars 2021. URL : http://journals.openedition.org/ bssg/345 ; DOI : https://doi.org/10.4000/bssg.345 
Enseigner la sociologie de la culture dans des formations professionnalisantes

Entretien avec Lionel Arnaud et Vincent Dubois

Frédéric Chateigner | Jérémy Sinigaglia
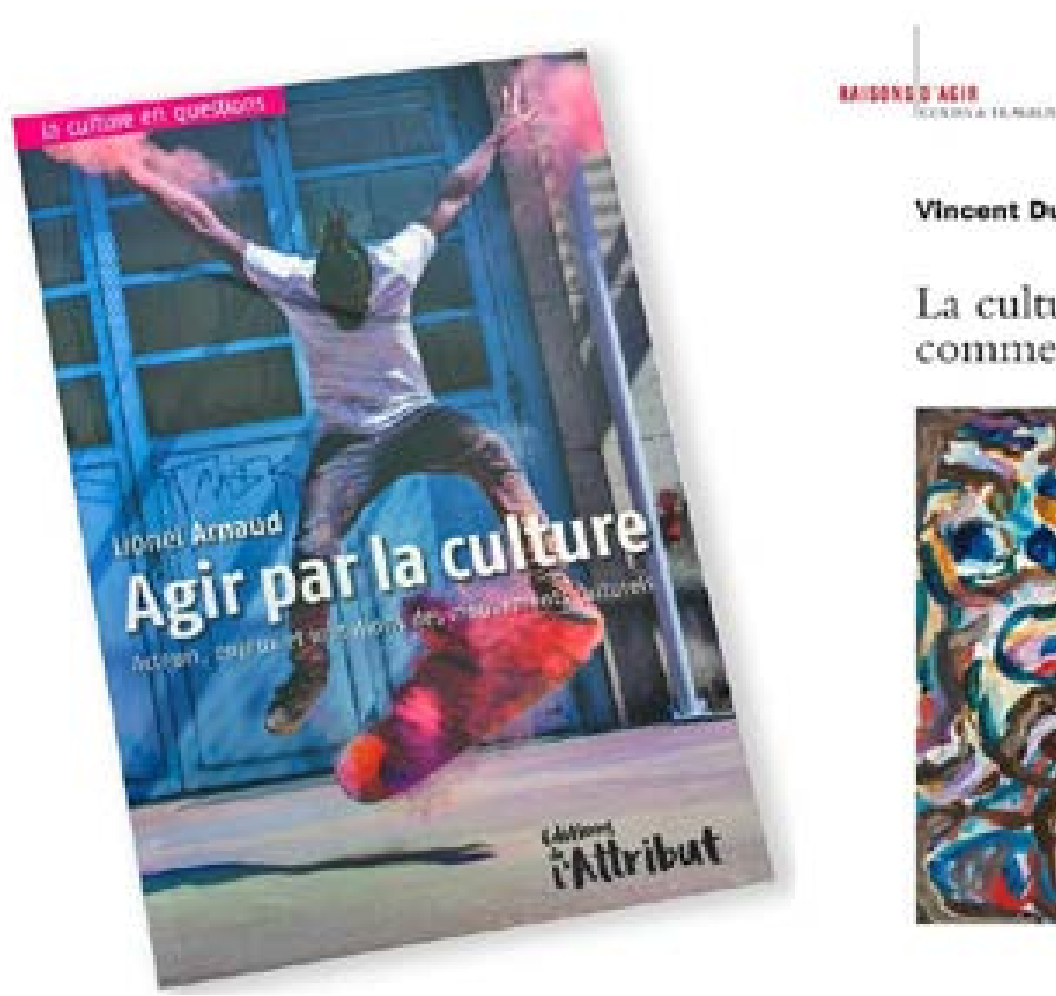

Vincent Dubois

La culture

comme vocation

Couvertures des ouvrages Agir par la culture et La Culture comme vocation

À gauche : première de couverture de l'ouvrage de Lionel Arnaud (2018).

Agir par la culture. Acteurs, enjeux et mutations des mouvements culturels. Toulouse, L'Attribut.

A droite : première de couverture de l'ouvrage de Vincent Dubois (2013).

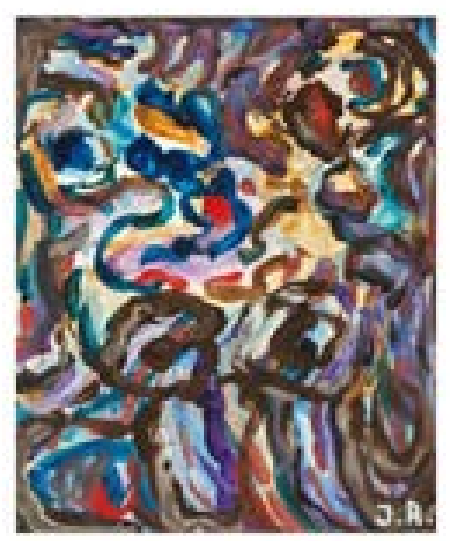

La Culture comme vocation. Paris, Raisons d'agir. 
Cet entretien vise à explorer quelques enjeux de l'enseignement de la sociologie de la culture dans des formations universitaires à visée professionnelle allant du diplôme universitaire de technologie (DUT) Carrières sociales jusqu'au master en passant par la licence professionnelle, aussi bien dans le management culturel ou sportif que dans l'animation socioculturelle. Nous avons pour cela réuni Lionel Arnaud et Vincent Dubois. Ceux-ci sont d'abord invités à présenter comment le montage de formations universitaires professionnalisantes s'inscrit dans leur trajectoire, puis à évoquer les collaborations avec le monde professionnel nécessaires à ces formations. L'entretien s'oriente ensuite vers les pratiques pédagogiques, auprès du public en formation continue ou en formation initiale, en particulier autour de la rupture avec les prénotions. On s'intéresse ensuite à la part de l'initiation à la recherche et à l'enquête de terrain dans cette pédagogie et à la difficulté de mesurer les effets de cette initiation. L'entretien souligne pour finir la façon dont les dispositions hétérogènes des publics étudiants envers les sciences sociales interrogent la posture même du sociologue.

\section{Trajectoires}

Les premières expériences de Vincent Dubois dans l'enseignement de la sociologie de la culture remontent au début de sa thèse de science politique à Lyon (Dubois 1994 ; 1999), avec des cours dans un des premiers master en gestion de projets culturels au début des années 1990. En 2004, il crée un master similaire à Strasbourg où il est devenu professeur.

Vincent Dubois (V. D.) : Déjà à cette époque il y avait énormément de formations dans ce domaine-là, et je me demandais jusqu'à quel point c'était vraiment judicieux d'en ajouter une énième, sur un marché de l'emploi qui était déjà relativement tendu. Mais j'y avais été invité en raison des politiques universitaires (développer l'offre en science politique par le niveau $M$ du tout jeune LMD, plutôt que par le niveau $L$ à la faculté de droit), mais aussi en raison de contacts que j'avais déjà noués, avec la Direction régionale des affaires culturelles (Drac), avec le Conseil de l'Europe qui a une politique culturelle assez active, et sur la base d'un constat local, qui était qu'il n'y avait pas du tout de formation de ce type dans ce qu'on appelle aujourd'hui le Grand-Est. Alors même que c'est une région, en tout cas pour l'Alsace, qui est très fortement dotée en structures culturelles, d'importance et de statuts différents, mais avec aussi des « besoins " en formation continue, qui étaient formulés comme tels par nos interlocuteurs.

Après une thèse en Staps sur les politiques d'intégration par le sport (Arnaud 1999), Lionel Arnaud a été recruté comme maître de conférences au département Carrières sociales de l'IUT de Rennes. En 2004, il coordonne le lancement d'une licence professionnelle en formation continue portée par ce département et par des associations d'éducation populaire, diplôme destiné à former aux fonctions d'encadrement intermédiaire dans le secteur de l'animation.

Lionel Arnaud (L. A.) : Ça a été une très belle aventure - le diplôme existe toujours d'ailleurs - notamment au niveau du travail de co-construction avec des intervenant.e.s professionnel-le.s qui, issu.e.s de l'éducation populaire, étaient non seulement très sensibles historiquement à la promotion par le savoir, la connaissance et au diplôme, et donc à la construction d'un type de formation qui pouvait créer des passerelles avec d'autres mondes 
et favoriser la montée en compétence d'un certain nombre de salarié·e.s et de bénévoles, mais aussi avec des professionnel·le·s de l'animation qui montraient en outre une formidable appétence intellectuelle à l'égard des sciences sociales, et une vraie réflexivité

Après une habilitation à diriger des recherches croisant les enjeux du sport, de l'animation et de la culture (Arnaud 2018), Lionel Arnaud est aujourd'hui professeur à l'université Paul-Sabatier de Toulouse, à la faculté des sciences du sport et du mouvement humain. II intervient aussi dans le master Études culturelles (Culture, média et politique) de Sciences Po Toulouse.

sur leur travail et leur identité. J'ai beaucoup appris avec eux.

\section{Monter un diplôme : quelles collaborations ?}

Jérémy Sinigaglia (J. S.) : J'aimerais que vous approfondissiez cette idée de "co-construction " des formations avec des professionnel/le.s du secteur culturel. On sait que c'est un terme en vogue dans le discours néomanagérial, et qu'on aimerait voir de la co-construction partout, mais concrètement : qu'est-il possible d'élaborer en commun et quelles difficultés cela pose ? J'imagine que les professionnel/le.s aident à identifier les «besoins " en compétence, mais que cela ne recoupe ni nécessairement ni complètement ce qu'un'e enseignant·e-chercheur'e souhaite et peut apporter. Comment se fait l'articulation entre, peut-être, ces deux manières de penser et de concevoir une formation universitaire?

V. D. : La création du master politique et gestion de la culture (PGC) a d'abord été faite conjointement avec une collègue de gestion, Odile
Paulus, puisqu'il apparaissait nécessaire pour justifier la création d'un master à vocation professionnelle de donner un certain nombre d'éléments en matière de gestion budgétaire, de constitution de dossiers de financement, de gestion des ressources humaines, etc. Donc il y avait déjà une alliance de ce côté-là et une ouverture sur des matières qui sont sans lien aucun avec mes propres orientations. Les relations avec les autres organismes de formation ont été relativement limitées. II y avait une structure qui n'existe plus aujourd'hui, l'Organisme de gestion des associations culturelles d'Alsace (OGACA), qui était l'homologue de l'Agence Rhône-Alpes de services aux entreprises culturelles (ARSEC) à Lyon. Au début des années 1980, le ministère de la Culture avait lancé, dans le processus de professionnalisation volontariste du monde de la culture, toute une série d'agences régionales, destinées à faire des études, à donner du conseil en gestion, voire à fonctionner comme structure gestionnaire, par exemple pour de petites troupes de théâtres, et certaines de ces agences avaient développé aussi de la formation. L'ARSEC s'était très fortement investie, avec un DESS qui avait ensuite été développé avec une maîtrise... À Strasbourg, l'OGACA proposait des formations à un niveau inférieur à celui que nous visions, du type "chargé de relations avec le public ", ou des formations davantage liées aux technicien.ne-s des métiers du spectacle. On était complémentaires et pas du tout concurrents mais on a intégré très vite un certain nombre d'intervenants de l'OGACA qui, eux, étaient spécialistes en gestion culturelle, des praticien-ne-s qui en plus avaient une pratique d'enseignement de ces matièreslà. Mais rien du côté des mouvements d'éducation populaire, des MJC, dans la mesure où cette formation était davantage ancrée dans le milieu de la culture avec un grand $\mathrm{C}$, correspondant plutôt au périmètre du ministère de la Culture.

Frédéric Chateigner (F. C.) : Ça s'est bien passé parce qu'il n'y avait pas de concurrence sur les fonctions et vertus de la culture? 
Comme si la place laissée à des intervenant.e.s extérieur.e.s, ou à des collègues d'autres disciplines, relevait d'une division $d u$ travail entre conception générale relevant des sciences sociales et des éléments techniques, "professionnels ", qui ne viennent pas marcher sur les plates-bandes de l'interprétation générale des universitaires.

V. D. : Effectivement ce n'est pas loin d'être ça. [...] Par ailleurs, à mi-chemin entre l'enseignement et la recherche, il y a aussi quelque chose comme la demande d'un regard extérieur, sous l'angle des recherches sur les pratiques culturelles ou sur les projets de développement des politiques de la culture. Ce sont des demandes d'institutions, de collectivités territoriales et je pense en particulier à la ville de Strasbourg avec un moment important qu'a été Les Assises de la culture, où le master et certain'e.s d'entre nous ont été partie prenante de ce qui se voulait être à la fois un vaste débat sur la place de la culture dans la ville et la collecte d'un certain nombre d'informations, de points de vue expert.e.s ou prétendu·e.s, sur les perspectives d'avenir des politiques de la culture au niveau local.

F. C. : Lionel, vous décrivez plutôt, presque à l'inverse, une relation quasi idyllique entre enseignant.e.s et professionnel-le·s : est-ce que ce n'est que ça ? Comment tient-on sa position de sociologue de tradition critique en rupture avec le sens commun dans ce contexte qui est effectivement séduisant, où on a l'impression qu'on va être à la fois sur la pratique et la théorie ?

L. A. : La construction d'une formation s'inscrit dans des dynamiques territoriales. De ce point de vue, je n'ai pas cherché au départ à monter une formation professionnelle : j'ai été avant tout porté par des dynamiques qui me dépassaient et que j'ai trouvé par ailleurs très positives en région Bretagne. Rétrospectivement, cela me paraît idyllique parce que je ne suis plus dans un contexte aussi porteur aujourd'hui. La Bretagne a une tradition historique plutôt favorable à l'éducation populaire qui a contribué à structurer les mouvements d'éducation populaire, à les rendre forts, et ça a favorisé une plus grande proximité entre les acteurs, avec des forces de proposition importantes. Mais il est vrai également que, quand on essaye de travailler, en tant qu'universitaire, dans le domaine de l'animation socioculturelle, on se heurte à des obstacles institutionnels, ne serait-ce parce que le DUT Carrières sociales option Animation concurrence directement les diplômes relevant du ministère Jeunesse et Sport. Les choses ont un peu évolué, des ponts commencent à se dessiner depuis, mais au tournant des années 2000 il y avait une vraie concurrence sur ce qui relevait déjà d'un véritable marché de la formation. De fait, les fédérations d'éducation populaire ne voyaient pas forcément d'un bon œil ces diplômes de l'enseignement supérieur qui, avec des droits d'inscription encore modiques, les concurrençaient sur leur propre terrain. Et ceci alors même qu'ils entendaient vendre et valoriser leurs propres formations, notamment aux diplômes d'État d'animateur. Donc on entrait dans des logiques qui ne sont pas seulement idéologiques, avec une éducation " populaire » qui entend se distinguer d'une éducation « nationale ", mais qui recouvrent aussi très clairement des positionnements d'ordre économique.

De ce point de vue, la présence à Rennes de l'Institut national de formation et d'études pour le développement (Infed) a permis de faire bouger les lignes en créant des passerelles entre d'une part le monde de l'université, avec le département Administration économique et sociale (AES) de Rennes 2 et l'IEP de Rennes, et d'autre part le monde des fédérations d'éducation populaire, et celui de Jeunesse et Sport. Et ces trois mondes, universitaire, associatif et administratif étant habitués, depuis des années, à se côtoyer en Bretagne, nous avons clairement bénéficié de cette dynamique, assez unique si on 
compare à d'autres territoires, pour monter avec l'IUT de Rennes la licence professionnelle Coordination de projet d'animation et de développement social et socioculturel, en 2004. Et puis nous avons profité de la création des licences pro, qui participait directement de ces logiques de professionnalisation qu'on connaît bien à l'université depuis une vingtaine d'années et qui visent à rendre les diplômes " utiles » et les étudiant·e.s « employables »... Moi en tout cas à cette époque, je l'ai vécu positivement, parce que ça a été un moyen de légitimer les savoirs et les outils des sciences sociales face à des praticien.ne's qui, il faut le dire, étaient aussi particulièrement demandeur·euse-s. L'histoire, la sociologie, la science politique, la géographie étaient ici mobilisés tout autant comme des instruments mis au service de la connaissance d'un public ou d'un territoire, que comme des outils de positionnements professionnels et politiques vis-à-vis de certaines questions sociales et culturelles : les inégalités sociales, les discriminations ethniques et raciales, le débat démocratisation/démocratie culturelle, la marchandisation des loisirs, le managérialisation des associations... II n'y avait donc pas à Rennes d'opposition trop marquée entre les universitaires et les animateurs et animatrices socioculturel'le·s. Ce n'est même pas qu'il y avait une division du travail bienveillante, mais une appétence de chaque côté pour ce que faisait l'autre, et donc la volonté d'articuler au mieux les savoirs des uns et des autres. Et puis les possibilités qui s'ouvraient alors étaient sans doute aussi liées à une génération d'animateurs et d'animatrices. Les personnes avec qui j'ai créé cette formation, qui avaient entre 45-60 ans à l'époque, avaient vécu toute une histoire militante de construction du domaine de l'animation qui s'est souvent faite en collaboration avec certain.e.s chercheur.e.s, à l'image de la revue Les Cahiers de l'animation qui, jusqu'en 1987 et sous l'égide de l'Institut national de l'éducation populaire (Inep, devenu depuis l'Injep), faisait se rencontrer des sociologues, des historien'ne-s et des praticien'ne-s (Lebon 2008). Aujourd'hui, il n'y a guère peut-être que l'Observatoire des politiques culturelles de
Grenoble qui continue à faire débattre de la sorte chercheur $\cdot e \cdot s$ et praticien.ne.s de la culture...

V. D. : Pour rebondir... Ce qui a présidé à la conception du master PGC, au-delà de ces alliances extérieures qui étaient très importantes, c'est aussi la conviction de l'intérêt des sciences sociales dans ce type de formation, fondée sur l'idée que la capacité critique qui peut être acquise grâce aux savoirs des sciences sociales, est en fait une compétence professionnelle nécessaire pour exercer des métiers dans ce secteur : savoir décrypter les jeux et les enjeux institutionalo-professionnels, aller gratter au-delà d'un discours souvent convenu, dont Franck Lepage a pu donner une illustration caricaturale (Lepage 2016), avoir un regard un peu aiguisé sur les questions centrales que sont l'inégale distribution des chances d'accès aux produits culturels, les questions de hiérarchie culturelle, de redistribution de ces hiérarchies, d'attitudes croisées légitimiste ou populiste... Tout cela, nous semblait-il, devait faire partie du bagage de quelqu'un'e qui prétend monter des projets culturels, qui est, au moins sur le papier, censé.e être intellectuellement construit·e. Donc l'idée c'était que la sociologie et le regard critique porté par la sociologie et plus généralement par les sciences sociales était vraiment un élément central de la formation, qui par ailleurs pouvait s'assortir d'enseignements beaucoup plus techniques.

\section{Enseigner la rupture avec les prénotions}

J. S. : Les étudiant.e.s semblaient-ils ou elles réceptif.ve.s à cette idée selon laquelle la critique serait pour eux·elles une compétence professionnelle utile ? Comment cela se joue-t-il dans les salles de classe, dans le rapport avec les étudiant $\cdot e \cdot s$ ?

V. D. : Je pense qu'il y a un facteur qui pouvait être important (en tout cas l'époque où j'y enseignais, les choses ont peut-être un petit peu 
changé) qui est que ce type de formation était aussi suivi, même si ce n'est pas majoritaire, par des personnes en reprise d'études, après avoir exercé plusieurs années comme animateur.trice-s

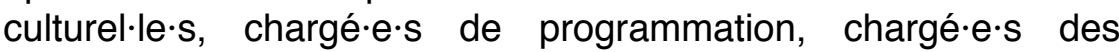
relations avec le public, etc. Ces personnes, pour des tas de raisons de promotion professionnelle, d'ouverture intellectuelle, voulaient revenir à l'université. C'est souvent un public qui est très friand de conceptualisation, d'un discours intellectuel qui leur permette de redonner du sens à leur pratique, de " sortir le nez du guidon », etc. Donc un public minoritaire dans ces formations, mais central dans l'animation des promotions, qui était vraiment en demande et assez en phase avec ce que l'on pouvait leur dire d'un point de vue sociologique, avec des prédispositions favorables à l'acceptation du discours sociologique. Alors évidemment, c'est un cas de figure, ce n'est pas le seul. II y a aussi les effets de déniaisement ou de désillusion et les effets aussi éventuellement de résistance à l'analyse sociologique qui sont présents dans ces milieux comme dans tous les milieux.

F. C. : Même si, comme le rappelait Vincent Dubois, les profils d'étudiant $\cdot e \cdot s$ en formation continue pèsent dans les promotions, ils elles sont minoritaires. Donc je voudrais que l'on parle aussi des étudiant.e.s en formation initiale, que ce soit en master, en licence ou en DUT, des publics très en demande de savoirs praticopratiques et qui peuvent avoir un rapport très naïf aux croyances dans les vertus de la culture, essentiellement artistique. Avec eux elles, comment fait-on? Est-ce qu'il faut rechercher ces effets de rupture, ou au contraire faut-il s'en méfier ?... Est-ce qu'il y a une boîte à idées de la rupture avec les prénotions?

J. S. : Pour prolonger un peu cette question, est-ce qu'on ne peut pas se demander si, en transmettant ces outils critiques, on ne se retrouve pas à fabriquer des étudiant.e.s soit cyniques, qui utiliseraient ces " armes » à des fins stratégiques, personnelles, soit malheureux euses, complètement désabusé.e.s?

V. D. : C'est compliqué. C'est vrai que si en tant que sociologue on a à cœur de montrer les choses sous un jour critique, il ne faut pas non plus se complaire dans ce qui pourrait être perçu comme le rôle du triste sire, qui dit que de toute façon, quoi qu'on fasse, ça ne sert à rien. II vaut mieux éviter de tomber dans une sorte de fatalisme qui éventuellement serait propice à une forme de désinvestissement, de désengagement, voire de renoncement y compris de ce à quoi malgré tout on croit, si je puis dire. C'est-àdire que c'est une bonne chose d'essayer de limiter les inégalités d'accès à la culture, d'essayer d'animer la vie culturelle et que ça fait partie intégrante de la vie sociale. C'est un équilibre qui n'est pas évident à trouver. L'un des premiers objectifs est simplement un rappel au réalisme. Pour le coup, la sociologie historique des politiques de la culture a aussi ces vertus : montrer comment une politique qui est conduite au nom de la démocratisation culturelle, quand elle se confond pratiquement avec une politique de l'offre culturelle, ne produit, par construction, pas les effets au nom desquels elle est conduite. Donc amener les étudiant·e-s à penser, y compris professionnellement, sous d'autres formes, autrement qu'en se disant « on va créer un nouveau festival et comme ça les gens pourront découvrir le jazz, la musique contemporaine ». Non, on sait que ça ne suffit pas, donc... Après, moi, je n'ai jamais donné de ficelles et de recettes pratiques, à l'issue de mes cours, mais de fait, en montrant ce qui marche plus ou moins, on peut dans les discussions avec les étudiant $\cdot$ e.s essayer de dégager des choses permettant au contraire d'être des leviers un peu plus efficaces.

Une des choses qui avait fonctionné assez bien, c'était une séance fondée sur les enquêtes qu'on avait faites sur les orchestres d'harmonie (Dubois, Méon, Pierru 2009), qui bouscule un petit peu 
la croyance relativement partagée que « les cultures populaires c'est bien, il faut les favoriser » mais en fait sans qu'on se demande de quel type de culture populaire il s'agit, et avec un impensé qui est que les cultures populaires qu'on promeut, ce sont celles qui ont l'heur de correspondre aux systèmes de goût et de jugement partagés par un certain nombre de professionnel-le·s, fondés sur des prédispositions sociales et des caractéristiques sociales. Là, en l'occurrence, c'est quelque chose qui d'un point de vue strictement professionnel n'avait pas vraiment d'incidence, car personne ne va travailler dans le monde des orchestres amateurs, des fanfares, etc. Mais montrer comment peut exister une forme de culture populaire relativement autonome, qui reste relativement active quasiment en dehors de toute aide publique liée à des perspectives de développement culturel, c'était de nature à faire se questionner les étudiant·e.s sur quels types de culture populaire ils ont en tête, qu'est-ce qu'ils elles peuvent finalement remettre en cause de leurs propres préférences implicites... « Voilà, la culture populaire c'est pour valoriser le rap dans les quartiers, pour amener les jeunes de quartiers à fréquenter les lieux de la culture légitime ", bon. Ce contrepoint, ancré dans le monde rural, très éloigné socialement et qui ne fait l'objet d'aucun discours d'accompagnement et de légitimation dans les milieux culturels, donnait une sorte de ligne de mire qui permettait en creux de déconstruire toute une série de présupposés sur, non pas : qu'est-ce que c'est apporter la culture au peuple ? Mais : comment valoriser les cultures populaires ?

L. A. : Mes recherches ne se sont jamais circonscrites à l'objet culturel au sens légitime, institutionnel du terme. J'ai toujours travaillé sur des objets plus variés : le sport et les activités physiques de loisirs, l'intégration des populations issues de l'immigration, le socioculturel, les villes et les politiques de développement social urbain... J'ai un peu tâtonné, ce qui n'est pas forcément très sécurisant dans le monde universitaire actuel, mais cela m'a en tout cas permis de ne pas rentrer dans des logiques académiques et institutionnelles trop circonscrites, notamment cette division du travail que l'on peut observer avec des masters qui, pour les uns, forment aux « métiers de la Culture » avec un grand C, quand les autres s'intéressent au reste, à l'animation socioculturelle ou aux pratiques sportives. Parce qu'on est ici dans des domaines fortement connotés et hiérarchisés symboliquement, et qui ne se parlent généralement pas. Les chercheur e.es qui travaillent sur ces différentes dimensions ne se rencontrent pas, ou alors rarement. Pour ma part, et pour des raisons variées, j'ai passé mon temps à me balader entre ces différents objets. Un tâtonnement qui a été en quelque sorte récompensé avec mon recrutement dans un département Carrières sociales option Animation sociale et socioculturelle, parce que l'animation par définition tend à se jouer des frontières, au moins en principe... Sauf que quand j'ai commencé à enseigner à Rennes, j'ai fait face à des étudiant·e.s qui attendaient avant tout qu'on leur dispense des savoirs pratiques. C'est une formation sur deux ans, donc ils elles sont censé.e.s être opérationnel·le·s au bout de deux ans. Mes premières années, je leur faisais des cours d'introduction à la sociologie, comme on me l'avait demandé lors de mon recrutement, mais je me suis rapidement aperçu que ça les assommait. Alors j'ai essayé de faire des cours de sociologie en phase avec les questions propres à l'animation et la culture. Mais qu'est-ce que «l'animation » et qu'est-ce que « la culture » ? Si l'on souhaite se départir des définitions institutionnelles et permettre aux étudiant·e.s de développer leur sens critique, il s'agissait évidemment de déconstruire ces objets en piochant dans la littérature scientifique. Force fut toutefois de constater que, en dehors des Cahiers de l'animation qui ont permis de mettre en débat intellectuel le sens de l'animation socioculturelle et de l'éducation populaire, il n'existait pas à proprement parler de travaux scientifiques susceptibles de nourrir une quelconque « sociologie de l'animation socioculturelle ". À cette époque, autrement dit au début 
des années 2000, je me suis donc heurté à une littérature historique ou sociologique qui portait soit sur les politiques culturelles, en particulier les travaux de Vincent Dubois, de Philippe Urfalino (1996), soit sur les politiques sportives, envisagées principalement sous l'angle de l'histoire des politiques d'éducation physique ou de l'olympisme coubertinien. J'étais donc condamné à faire des cours soit sur l'un soit sur l'autre, comme ça se fait dans certains IUT. Sur l'éducation populaire, il y avait bien quelques articles ou ouvrages mais ils étaient généralement assez datés : pas grand-chose sur I'histoire récente, alors même que l'on assistait à une sorte de regain du mot « éducation populaire » dans le discours militant. Ma stratégie a d'abord été celle d'une reconstruction sociohistorique, en puisant certes dans ces différents travaux mais pour faire les liens que leurs auteurs ne faisaient pas. Et encourager ainsi les étudiant·e.s à questionner ces champs d'intervention publique qui, tels la « culture » et le « sport », contribuent à enfermer les pratiques sociales et culturelles que les animateurs entendaient précisément « émanciper ». Pour les aspirant·e·s aux métiers de «l'animation», du « sport » et de la « culture » c'est évidemment un exercice utile parce que ça leur permet de dénaturaliser leur rapport à leur objet. De montrer aussi que les débats qu'il peut y avoir aujourd'hui sur " la culture » et le rôle de son ministère, ou les reconfigurations qui se développent au niveau territorial sont en fait très anciens en France... Cela permet de montrer comment se sont constitués de manière très idéologique des positionnements professionnels dont ils et elles sont aujourd'hui les récipiendaires... À ce niveau, et dans cette même logique de mise en comparaison des champs d'intervention mais aussi des univers culturels, la recherche que j'ai conduite en Martinique à partir de 2011 m'a énormément apporté, dans la mesure où c'est un territoire dont l'histoire, les expressions culturelles et tout simplement la population ont été tellement marginalisées que les hommes et les femmes qui y vivent n'ont eu d'autres choix que d'y développer leur propre action culturelle, ou plutôt une « contre action culturelle ", en s'auto-organisant dans un monde où les institutions culturelles avaient moins d'emprise que de ce côté-ci de l'Atlantique. Et donc quand je fais découvrir aux étudiant·e.s des pratiques culturelles comme le danmyé, qui n'est ni un sport, ni une danse, ni un art, ni une pratique spirituelle, ni une pratique politique... mais tout ça à la fois, c'est souvent une bonne manière d'interroger les stratégies institutionnalisantes consistant à sportiviser ou à esthétiser les pratiques culturelles, à l'image de ce qui s'est produit dans les années 1980-1990 avec le mouvement hip-hop... Leur montrer des vidéos de cette sorte de " capoeira martiniquaise ", c'est aussi une manière d'interroger les raisons qui expliquent que le danmyé soit quasi-inconnu en France alors que la capoeira y est ultra-célèbre et diffusée... L'usage de la comparaison nationale et internationale, le détour par d'autres univers, d'autres pays, d'autres cultures, m'est souvent apparue comme une façon efficace de bousculer les évidences et la bonne volonté culturelle des futur.e.s « professionnel-le.s de la culture et du sport ».

\section{L'initiation à la recherche}

J. S. : Quelle peut être ou doit être la place, selon vous, de la recherche, l'initiation à une pratique un peu minimale de la recherche, dans ce type de formations ? Dans la maquette du master PGC, pendant longtemps il y a eu un mémoire obligatoire, maintenant la formation à et par la recherche passe plutôt par des enquêtes collectives plus orientées... il semble que, dans les deux cas, ce soit une façon pratique de confronter les étudiant.e.s aux catégories, et de les faire participer à l'effort de déconstruction.

V. D. : Dans cette conception, il y avait l'idée que le mémoire puisse être une occasion personnelle et pédagogique de formation intellectuelle : tout professionnel qu'il est, ce master est une formation universitaire. Le mémoire peut être un travail 
de type sociologique comme il peut relever d'autres disciplines, les croiser, ou avoir une dimension plus directement pratique. Le simple fait d'être confronté à la construction d'une analyse, d'un texte, oblige à se confronter à un certain nombre de choses qui peuvent être considérées comme allant de soi. Bon ça, c'était les intentions (rires). Parfois cela a très très bien marché d'un point de vue académique, c'est-à-dire que le master et le mémoire ont fonctionné comme des éveils à la vocation de chercheur.e, avec des gens qui ont continué en thèse. Ce n'est pas le but premier, c'est marginal, mais ça a existé. II y a eu parfois des très très bons travaux d'un point de vue strictement scientifique. II y avait aussi, il faut bien le dire, beaucoup de travaux moins intéressants, un peu bâclés parce que les étudiant·e.s le font quand ils·elles font un stage sur un tout autre domaine. Et c'était parfois considéré, à tort à mon avis, un peu comme un pensum académique plutôt que comme une occasion à la fois personnelle et professionnelle de réfléchir à l'univers dans lequel ils elles vont travailler. D'où la refonte récememnt engagée dans ce master avec l'instauration d'un travail moins lourd, moins exigeant pour les étudiant $\cdot e \cdot s$, sans pour autant perdre ce rôle réflexif.

L. A. : Pardon d'être un peu prosaïque et de nous ramener à des logiques et des contraintes institutionnelles, mais il y a ce qu'on voudrait faire et ce que l'on peut faire. II faut rappeler que dans les masters il n'y a plus officiellement de parcours recherche, les heures dévolues à la formation par et à la recherche sont donc de plus en plus difficiles à trouver et c'est devenu compliqué de maintenir ce type de formation (et de vocation) dans des masters qui se présentent avant tout comme des formations " professionnelles ", avec tout ce que cette terminologie comporte de sens plus ou moins caché... Alors on bricole. Par exemple en invitant l'étudiant·e à mobiliser les méthodes des sciences sociales sur son terrain de stage. Cela l'oblige à avoir une réflexion sur ses pratiques professionnelles et celles des autres, tout en mobilisant les outils de la recherche qui relèvent de l'enquête ethnographique et de la participation observante. C'est un compromis, pas forcément satisfaisant, mais qui a ses vertus, notamment parce qu'il contribue à former les étudiant $\cdot e \cdot s$ à des méthodes de recherche encore trop peu valorisées.

\section{Quels effets ?}

J. S. : C'est très bien d'essayer de penser nos pratiques pédagogiques, mais a-t-on un moyen d'en mesurer un peu les effets ? Comment peut-on essayer de savoir si ça passe ou pas, s'ils.elles arrivent à s'approprier les outils que l'on essaye de transmettre?

V. D. : C'est très compliqué... notamment parce que l'année est trop courte pour mesurer des effets. Cela peut passer par les moments de soutenance, effectivement. Un indicateur peut-être, ce sont les témoignages a posteriori quand je recroise des étudiant $\cdot e \cdot s$ quelques années plus tard : ils elles me disent que, pendant leur formation, l'intérêt de tel ou tel cours, voire des sciences sociales en général, n'était pas évident, jusqu'à ce que, confronté·e·s à telle situation, ils elles le comprennent soudain. Ce n'est pas quotidien mais cela arrive.

L. A. : Oui, idem pour moi. C'est parfois lors des soutenances que l'on réalise que l'on a fait du chemin. Lors de mes suivis de mémoire, je fais travailler les étudiant·e.s sur un journal qui est à la fois un journal d'enquête, un journal intime et un journal de recherche. J'ai repris ça de mes collègues issu.e.s des sciences de l'éducation et du travail social à I'IUT de Rennes, et de ma propre expérience de chercheur. Pour celles et ceux qui jouent le jeu, c'est devenu un précieux instrument de réflexivité et puis 
ca permet de savoir comment leur positionnement évolue. Sans compter bien sûr l'intérêt de ce type de journal du point de vue de l'écriture, du développement d'une certaine sensibilité à l'égard de leurs environnements humains et matériels. Mais pour le reste, on touche je crois aux limites des évaluations auxquelles sont soumises nos formations : savoir si nos formations sont " utiles" ou « efficaces », si nos étudiant·e·s sont " employables ", s'ils elles ont trouvé du travail... Une formation, quelle qu'elle soit, se joue forcément à long terme, ce n'est pas forcément très spectaculaire ce qu'une formation peut produire. Du coup, je n'ai pas vraiment de réponse. Sauf effectivement le témoignage d'ancien'ne-s étudiant $\cdot \mathbf{e} \cdot \mathrm{s}$, mais faut-il encore les revoir. Attention en tout cas au piège qui consisterait à tout mesurer, à tout évaluer. On le sait, il y a plein de choses qui ne s'évaluent pas et qui s'incorporent sans crier gare.

J. S. : D'ailleurs, le fait que les étudiante.s comprennent, et restituent correctement ce qui est attendu d'eux.elles dans une évaluation, n'est pas nécessairement une preuve de cette incorporation. Il est difficile de distinguer ce qui relève de la bonne réponse à un exercice académique de ce qui a vraiment agi sur les principes et les catégories de vision du monde.

V. D. : Oui, nous parlions tout à l'heure des formes de résistance. II me semble - mais là il faudrait faire vraiment une sociologie de la réception des différents enseignements - que ce ne sont pas nos enseignements qui suscitent forcément le plus de résistance et d'opposition. J'ai des souvenirs d'oppositions frontales et houleuses à l'égard de cours de type marketing, en l'occurrence dispensés par un type qui ne brillait pas par sa délicatesse et qui commençait son cours en disant : " la culture ça se vend comme des pots de yaourts ". On a d'une certaine manière des étudiants.e.s qui sont peut-être plus perméables au discours critique de la sociologie avec les implications politiques que ça peut avoir, qu'ils elles ne sont perméables au discours cynique d'une certaine forme d'enseignement managérial ou en termes de marketing. En même temps, c'est souvent ces savoir-faire pratico-techniques valorisables sur un $\mathrm{CV}$ et pour l'insertion professionnelle que ces étudiant·e.s viennent chercher. Mais c'est aussi, en fonction de la façon dont c'est enseigné, ceux qui suscitent le plus concrètement de rejet.

\section{Dispositions hétérogènes et posture sociologique}

L. A. : Mais est-ce que ce n'est pas aussi un effet de position, qui fait que les étudiant·e.s que vous avez, qui sont plutôt en filière sciences po, sont déjà sensibles à un discours critique ? C'està-dire que les formations au management de la culture qui sont dispensées à Dauphine ou dans des écoles de commerce, sont peut-être beaucoup plus...

V. D. : Bien évidemment, il faudrait distinguer. Même si rien ne dit que les étudiant.e.s d'IEP sont plus critiques. En master PGC nous n'avions pas essentiellement des étudiant.e.s de sciences po : beaucoup venaient d'autres cursus, dont pas mal de parcours artistiques.

L. A. : Quand je compare par exemple les étudiantees que j'ai en master Études culturelles à sciences po, il y a une réceptivité assez évidente aux sciences sociales, et notamment à la sociologie critique. On peut même dire qu'ils et elles y sont biberonné.e.s ! En DUT Carrières sociales et en licence pro, c'était moins la sociologie critique qui intéressait les étudiant.e.s que les sciences de l'éducation, les courants et les techniques pédagogiques, envisagés comme des outil de lutte et d'émancipation... La philosophie 
critique de Jacques Rancière est par ailleurs très populaire parmi les professionnel-le.s de la culture et les artistes que je rencontre, sans doute parce qu'elle est moins désespérante que la sociologie de Pierre Bourdieu ! Et qu'elle permet de conforter et de légitimer le travail et les croyances de ces acteur·trice-s. Les étudiant·es de M2 management du sport, pour leur part, en ont franchement ras la casquette des sciences sociales ! lls elles le disent d'ailleurs clairement... ce qu'ils elles veulent ce sont des cours de gestion, de marketing, de communication... normal, puisque c'est ce que leurs employeurs attendent...

F. C. : Dans les filières que vous évoquez, il y a une diversité du public : il y a à la fois des gens qui, sur la base d'une formation artistique notamment, sont attachés à la défense d'une universalité et de l'autonomie de la création, et qui vont aller vers - on ne va pas dire un cynisme, mais, disons - un certain réalisme économique via le management de la culture ; mais il y a aussi, à l'inverse, des gens qui viennent de formations beaucoup plus orientées vers le monde économique, et qui vont chercher un supplément d'âme. C'est le caractère de "zone frontière » de ces formations de management des biens universels, peut-être, qui explique cette diversité... C'est une hypothèse possible. Or, je me demande si nos disciplines - sociologie, science politique, histoire - n'ont pas un peu le beau rôle dans ces formations-là, parce qu'on peut toujours jouer les deux côtés à la fois : le cynique avec les idéalistes et l'idéaliste avec les cyniques, en quelque sorte.

V. D. : Sur le premier point, je serais d'accord, je l'évoque à la fin de La Culture comme vocation (Dubois 2013) : il y a un nouvel esprit du capitalisme incarné dans les trajectoires croisées que vous évoquez. Une combinaison entre l'incorporation de la critique artiste du capitalisme, et en même temps la référence à la nécessité du management qui, bien qu'elle puisse engendrer des réactions parfois hostiles, est assez largement intégrée. Sur le deuxième point : je n'y avais jamais pensé en ces termes mais nous n'avons pas tellement d'occasions d'assumer une position confortable!

L. A. : Cela me fait penser à deux anecdotes récentes. Une personne est venue me voir spontanément, qui travaille au CCAS, le comité d'entreprise d'EDF, et qui s'est inscrite en formation continue à l'université Paris-Dauphine au sein du master Management des organisations culturelles. Ce qui n'a pas manqué de m'étonner, d'une part parce que c'est une formation éloignée de Toulouse alors même que nos universités locales proposent des diplômes quasiidentiques. Et puis parce que le CCAS est géré par la CGT, un syndicat a priori en pointe de la critique sociale. Elle m'a dit « oui, mais moi le directeur du CCAS il veut surtout que j'aie des outils techniques, des outils gestionnaires et financiers ", sous-entendu le reste ça l'intéresse pas trop. Deuxième anecdote : une amie qui est responsable d'un théâtre plutôt militant à Lyon m'a appris, elle, récemment qu'elle avait choisi de suivre ce même master à Dauphine. II ne s'agit pas ici de diaboliser le savoir managérial - ne serait-ce que parce qu'il constitue aujourd'hui, et quoi qu'on en pense un savoir-faire indispensable - pour trouver des financements, voire tout simplement vivre de son art... Mais dans ces deux exemples, ces personnes voyaient avant tout l'intérêt d'aller en formation pour acquérir des outils de management, tandis que les savoirs ancrés dans les sciences sociales leur semblaient secondaires... Comme si, en leur qualité de militantes de longue date, elles n'avaient rien à apprendre de ce côté-là. C'est pour cela je pense qu'il nous faut reprendre la main, dans un contexte où les formations universitaires se « déscolarisent » au profit d'une pensée pragmatique et anti-intellectuelle. Quand on parle de « sociologie critique ", on pense trop souvent à l'intellectuel-le militant $\cdot e$, voire révolutionnaire, celui ou celle qui va vous apprendre la vie, avec une posture un peu hautaine, quasi missionnaire... celui ou celle 
qui, du haut de son savoir et de ses diplômes, va vous dire quoi faire et quoi penser. Ça donne une image de donneurs de leçons, en retrait de "la vraie vie ", des « vrais gens ", à une époque où les élites intellectuelles n'ont peut-être jamais été autant décriées. On a ici un gros problème d'image publique, qui empêche certains savoirs d'être diffusés au plus grand nombre et qui ouvre finalement la voie à une pensée de l'immédiat, celle des webographies que je trouve désormais systématiquement à la fin des mémoires de M2. II nous revient donc de mieux valoriser le sens même de la critique, scientifique, celle qui permet de mettre à distance les approches dominantes et donne à voir d'autres rapports à la réalité.

Ce qui suppose aussi d'avoir des prises de parole en dehors de l'université, et de prendre en compte les savoirs et les savoir-faire des praticien'ne·s, leur manière de voir et de saisir la réalité, sans les renvoyer systématiquement à leurs supposés aveuglements, à leurs dispositions et à des logiques qui les dépasseraient. Alors certes, la recherche en sciences sociales a depuis une vingtaine d'années pas mal évolué à ce niveau-là, notamment grâce à la diffusion des méthodes d'enquête ethnographique qui donnent un poids plus important à la parole des « dominé·e.s ». Mais il nous faut encore lutter contre cette image du chercheur ou de la chercheuse « hors sol »... En disant cela, je pense toujours à Bourdieu qui, dans le film documentaire de Pierre Carles La Sociologie est un sport de combat, dit à des habitant.e.s du Val Fourré (quartier de Mantes-la-Jolie) : " Je vous connais mieux que vous-mêmes. " C'est certes fondé scientifiquement mais l'expression n'en est pas moins dévastatrice pour l'image des sociologues, et des intellectuel·le·s en général, dans la mesure où, en objectivant leurs positions sociales, cette déclaration tend à renforcer finalement la domination des publics profanes. Or, si on braque les populations les plus éloignées des sciences sociales en les prenant pour des idiots sociaux, on aura toujours plus de mal à les impliquer dans une réflexion sociologique qui serait au principe d'une meilleure intelligence des mécanismes de domination.
Frédéric Chateigner

Université François-Rabelais, Tours (IUT, département Carrières sociales)/ Laboratoire Sociétés, acteurs, gouvernement en Europe (Sage)

\section{Jérémy Sinigaglia}

Université de Strasbourg - Laboratoire Sociétés, acteurs, gouvernement en Europe (Sage)

\section{Références bibliographiques}

ARnAUD Lionel (1999). Politiques sportives et minorités ethniques. Le sport au service des politiques sociales à Lyon et à Birmingham. Paris, L'Harmattan.

ARNAUd Lionel (2018). Agir par la culture. Acteurs, enjeux et mutations des mouvements culturels. Toulouse, L'Attribut.

DuBoIs Vincent (1994). La Culture comme catégorie d'intervention publique. Génèses et mises en forme d'une politique. Thèse de doctorat en science politique. Lyon, Université de Lyon 2.

DuBoıs Vincent (1999). La Politique culturelle. Genèse d'une catégorie d'intervention publique. Paris, Belin.

DuBols Vincent (2013). La Culture comme vocation. Paris, Raisons d'agir.

DuboIs Vincent, MÉON Jean-Mathieu, PIERRU Emmanuel (2009). Les Mondes de l'harmonie. Enquête sur une pratique musicale amateur. Paris, La Dispute. 
LEBON Francis (2008). " Des intellectuels sécants ? Enquête sur les auteurs des Cahiers de l'animation (1972-1987) ". Un engagement a l'épreuve de la théorie. Itinéraire et travaux de Geneviève Poujol. Paris, L'Harmattan : 71-91.

Lepage Franck (2016). «Incultures 1 : L'Éducation populaire monsieur, ils n'en ont pas voulu ». [En ligne] [consulté le 15 janvier 2018].

UrfaLino Philippe (1996). L'Invention de la politique culturelle. Paris, La Documentation française. 